\title{
Cystic hepatic mesenchymal hamartoma: the role of radiology in diagnosis and perioperative management
}

\author{
${ }^{1,2} \mathrm{G}$ ANIL, MD, FRCR, ${ }^{1} \mathrm{M}$ FORTIER, MD, FRCP and ${ }^{3} \mathrm{Y}$ LOW, FRCS, FAMS

\begin{abstract}
${ }^{1}$ Department of Diagnostic Imaging, K K Women's and Children's Hospital, 100 Bukit Timah Road, Singapore 229899,
${ }^{2}$ Department of Radiology, Changi General Hospital, 2 Simei Street 3, Singapore 529889, and ${ }^{3}$ Department of Paediatric Surgery, K K Women's and Children's Hospital, 100 Bukit Timah Road, Singapore 229899
\end{abstract}

\begin{abstract}
A 13-month-old male presented with large hepatic mass that was confirmed diagnostically to be a cystic hepatic mesenchymal hamartoma. The sieve-like appearance of the solid components of the mass, as seen at ultrasonography, and findings of dynamic post-contrast MRI are highlighted here. Beyond diagnostic imaging, we extended our role in patient management through ultrasound-guided intra-operative aspiration of fluid from the cystic components of the tumour to reduce its volume and thereby facilitate surgical resection.
\end{abstract}

Received 29 March 2010

Revised 27 June 2010

Accepted 15 July 2010

DOI: $10.1259 / \mathrm{bjr} / 41579091$

(C) 2011 The British Institute of Radiology
Mesenchymal hamartoma $(\mathrm{MH})$ of the liver is a rare, benign developmental malformation composed of a mixture of loose mesenchymal tissue, bile ducts, connective tissue and hepatocytes along with cysts formed either from degenerative areas of mesenchyme or from dilated bile ducts and lymphatics [1, 2]. They are predominantly cystic, with the stromal predominant type rarely seen [1]. This pathology was variably labelled as lymphangioma of the liver, bile duct fibroadenoma, cavernous lymphangiomatoid tumour, giant lymphangioma and pseudocystic mesenchymal tumour before being unified under the single term "mesenchymal hamartoma". This term conveys that they are not true neoplasms.

We present a case that demonstrates an approach from clinical presentation to treatment of $\mathrm{MH}$ with an emphasis on dynamic post-contrast MRI and ultrasound appearance. We also highlight the scope for radiologists to expand their role in peri-operative patient management, through the example of the simple but pro-active step of ultrasound-guided intra-operative aspiration of fluid from the massive cystic components of the mass that facilitated surgical excision in this patient.

\section{Case report}

A 13-month-old male presented with abdominal distension, shortness of breath and loss of appetite for the past 2 months. On examination, non-tender abdominal mass was noted. Besides elevated serum alphafetoprotein (830 IU; normal 0-10) and gamma-glutamyl transpeptidase (127 $\mathrm{U}^{-1}$; normal $<18 \mathrm{U}^{-1}$ ), the laboratory reports were unremarkable. There were no signs

Address correspondence to: Dr G Anil, Associate consultant, Department of Radiology, Changi General Hospital, 2 Simei Street 3, Singapore 529889. E-mail: ivyanil10@gmail.com of sepsis. The patient underwent an ultrasound of the abdomen on the day of admission and an MRI of the abdomen on the following day. A 1-month-old CT scan, performed elsewhere, was reviewed.

Ultrasound revealed a well-defined, multicystic mass involving the right lobe of liver. The cysts were anechoic and variable in size (largest $12 \mathrm{~cm})$. Thin $(1-2 \mathrm{~mm}$ ) intervening septae and peripheral solid components were noted. The solid areas were isoechoic to the liver but demonstrated a sieve-like appearance constituted by multiple tiny (1-4 mm) anechoic areas with posterior enhancement and intervening iso- to hyperechoic tissue (Figure 1). Colour Doppler interrogation showed minimal vascularity. The visualised portal and hepatic veins in the vicinity were displaced. There was no biliary dilatation or evidence of portal hypertension. The review of postcontrast CT images showed a circumscribed hypodense mass with minimally enhancing septations and peripheral solid components but no internal calcification. There was no ascites, lymphadenopathy or cystic masses elsewhere in the abdomen.

MRI of the liver was performed under general anaesthesia on a 1.5 Tesla scanner using multiplanar $T_{1}$ weighted $\left(T_{1} \mathrm{~W}\right)$ (dual echo spoiled gradient recalled acquisition in steady state (SPGR), three-dimensional (3D) liver acquisition with volume acquisition (LAVA), pre- and dynamic post-contrast with delayed images), $T_{2}$ weighted $\left(T_{2} \mathrm{~W}\right)$ (fast spin echo (FSE), single shot fast spin echo (SSFSE), fast imaging employing stead state acquisition (FIESTA)) and oblique axial time-of-flight sequences. Most of the images were obtained using breath-hold sequences. The well-defined, non-capsulated mass measured $16 \times 11.4 \times 12 \mathrm{~cm}$ in size. Although predominantly cystic, MRI revealed more solid areas than those seen on ultrasound. Compared with normal liver, the solid areas and septae were hypointense on $T_{1} W$ and hyperintense on $\mathrm{T}_{2} \mathrm{~W}$ images. The cystic areas showed homogeneous fluid 


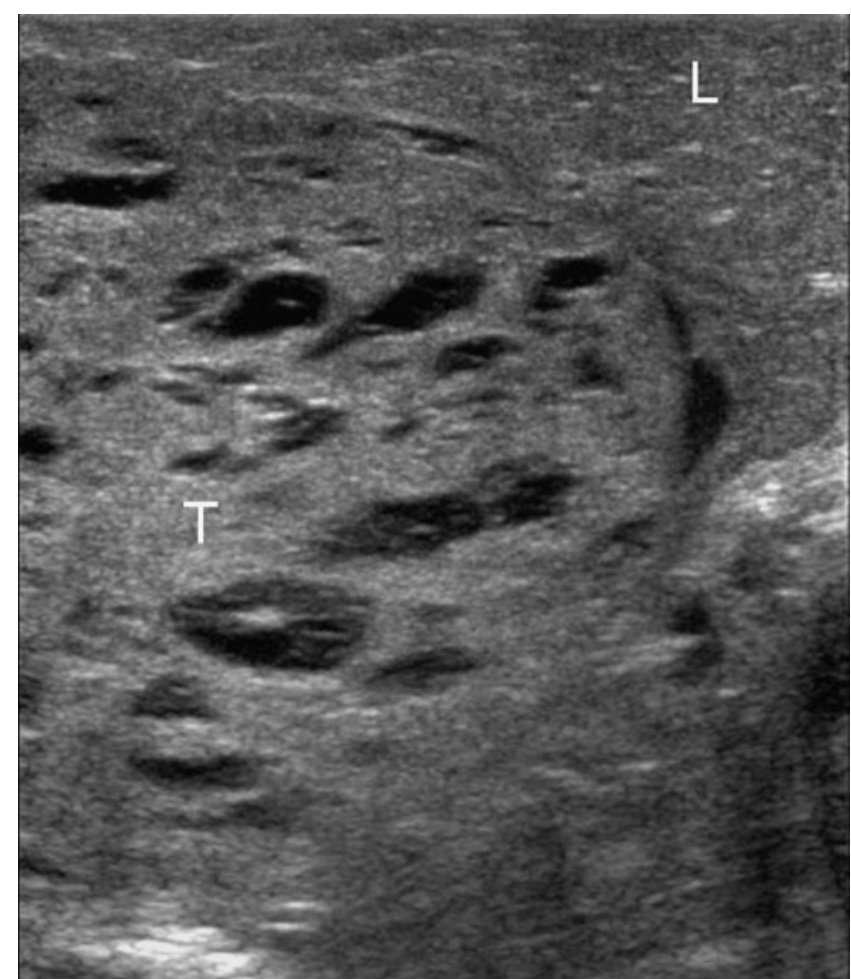

Figure 1. This high resolution ultrasound image shows the sieve-like appearance of the solid components (T) of the hepatic mesenchymal hamartoma; otherwise the solid areas are almost isoechoic to normal liver (L). Note the sharp outline between the mass and normal liver.

signal (Figure 2). The post-gadolinium images showed almost no enhancement in the arterial phase with mild enhancement in the porto-venous and delayed phase images. The tumoural enhancement was less than normal liver in every phase of post-contrast imaging (Figure 3). The right hepatic vein was not visualised. The middle and left hepatic veins, hepatic segment of inferior vena cava (IVC), the portal vein and its right branch as well as the right hepatic artery were displaced, with no obvious infiltration, encasement or thrombosis.

Within 4 days of the MRI of the abdomen, under general anaesthesia, ultrasound-guided fluid aspiration was performed to exclude hydatid disease. Subsequently, core-biopsy was performed from the solid components of the mass in the same sitting. Histopathological evaluation diagnosed MH. 2 days later, elective right hepatectomy for complete resection of the mass was performed.

During the hepatic resection, the surgical team requested an intra-operative ultrasound (IUS). In collaboration with the operating surgeon we performed ultrasound-guided aspiration of fluid from several large cysts using an $18 \mathrm{G}$ needle (Figure 4 ). The consequent marked reduction in tumour volume decreased the mass effect on the displaced vessels, enabling confident IUS evaluation for their patency. Adequate margins for dissection, separate from the vessels, were found and the tumour was completely excised. The surgery and convalescence were uneventful. Histopathology of the surgical specimen confirmed the diagnosis of cystic $\mathrm{MH}$.

\section{Discussion}

$\mathrm{MH}$ is the second most common benign paediatric liver tumour ( $5 \%$ of primary liver tumours) after haemangioma $[3,4]$. Yet it is rare enough to be seen only once in 2 years even at major centres [5]. Although benign, occasional malignant degeneration into undifferentiated embryonal sarcoma is known [4]. In a series of 30 patients, $\mathrm{MH}$ was found predominantly in the right lobe of liver (right: left, 6:1), especially in males (male: female, 2:1) younger than 2 years of age [2]. Cases have been reported in foetuses, older children and adults. Usual presentation is a rapidly enlarging, painless, abdominal mass with an average size of $16 \mathrm{~cm}$ [1]. Alpha-fetoprotein may be elevated in some patients with $\mathrm{MH}$, as seen in our patient [6]. However, elevated gamma-glutamyl trans-peptidase, with the rest of the liver function tests normal, is unusual; we could not find a similar observation in the published literature. Imaging studies have a major role in patient management, both in diagnosing $\mathrm{MH}$ as well as in defining the tumour extent and relationship that informs the feasibility of and approach to surgery. Enucleation, marsupialisation of cysts, excision of hamartoma with surrounding rim of normal liver tissue and formal hepatic lobectomy are the prescribed treatment options [4].

Based on ultrasound and CT characteristics, MH has been described in several case reports and short series. As $80 \%$ of $\mathrm{MH}$ have macroscopic cysts [2], they are usually seen as a large multiloculated cystic masses with

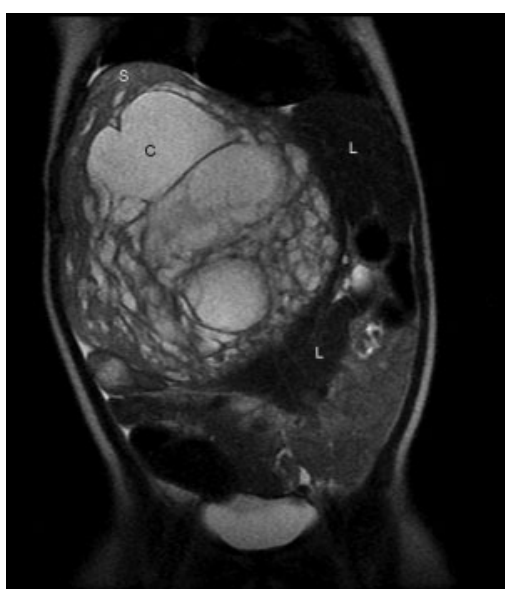

(a)

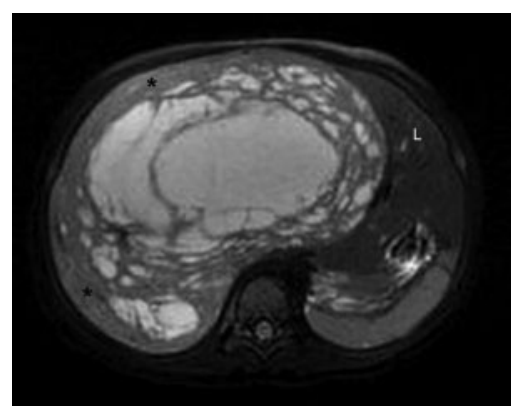

(b)
Figure 2. These (a) coronal and (b) axial $T_{2}$ weighted images exquisitely depict the massive size of the mesenchymal hamartoma with multiple cystic areas (C) of varying sizes, few solid areas (S) and internal septations. The solid areas are hyperintense to normal liver (L). 


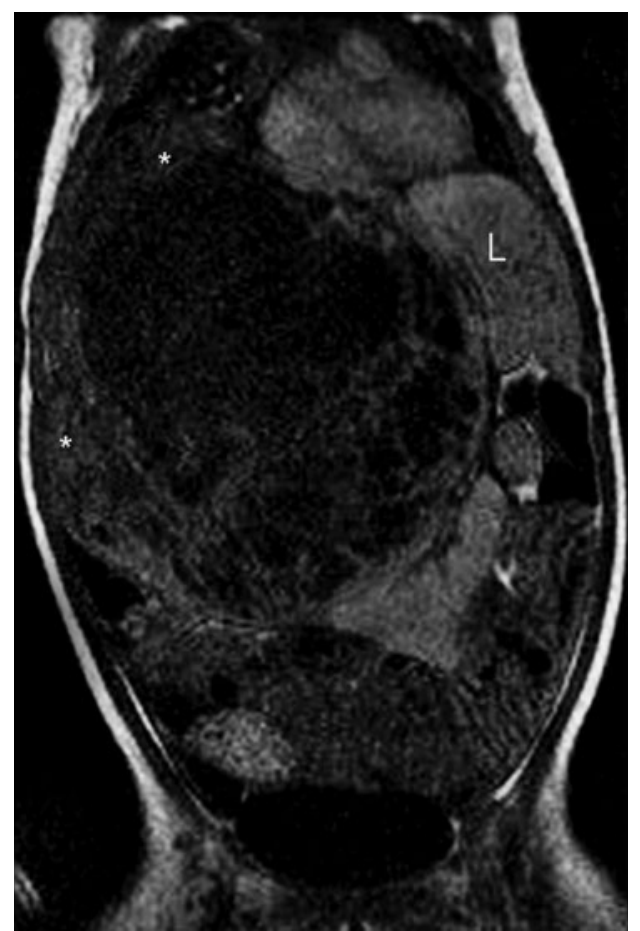

(a)

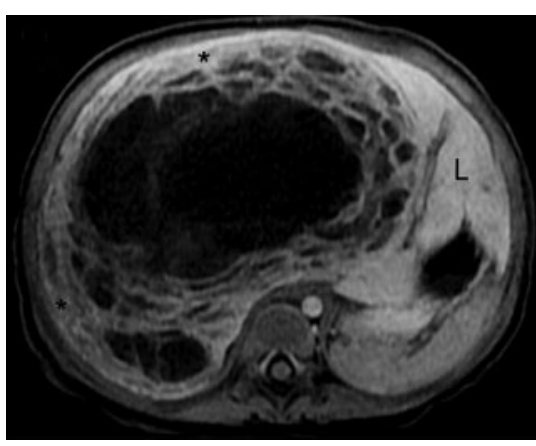

(b)

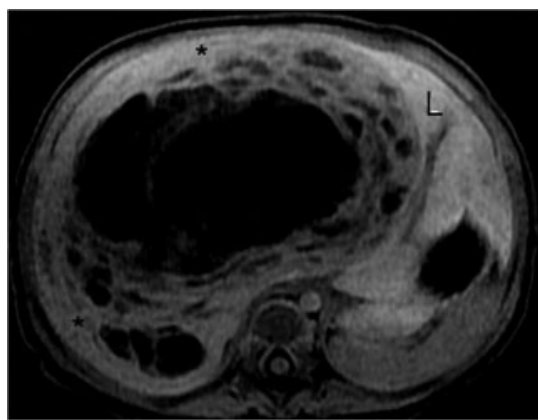

(c)

Figure 3. These are the post-gadolinium $T_{1}$ weighted (a) arterial phase, (b) portovenous and (c) delayed phase images. There is almost no enhancement seen in the arterial phase while poor enhancement is seen in the MRI images obtained in the portovenous and delayed phases (asterisk, solid areas of the mass; L, normal liver).

internal septations. Both ultrasound and MRI depict the cysts and septae well, as noted in this case report. At high frequency ultrasound, we found a sieve-like appearance of the solid components of the tumour. This is probably the ultrasound equivalent of the Swisscheese appearance that is seen on gross pathological examination with the pores of the sieve representing the tiny cystic spaces. On CT it is seen as a low attenuating tumour with or without septations $[1,6,7]$. CT may not always show the septae, and hence differentiating the cysts of $\mathrm{MH}$ from the heterogeneous necrotic areas as

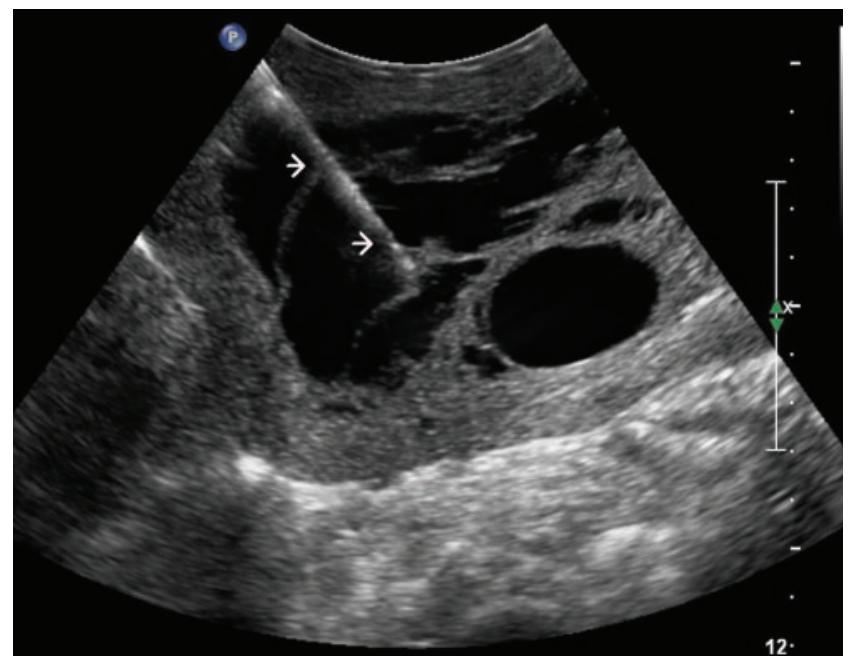

Figure 4. This intra-operative ultrasound image shows the needle (arrows), in one of the larger cysts of the mesenchymal hamartoma. well as hypovascular/hypoattenuating regions within an aggressive malignant neoplasm may be difficult; however, ultrasound or MRI correlation may often be useful in this regard as they enable better demonstration of cystic areas [7]. $\mathrm{MH}$ is hypovascular [1, 7] with mild enhancement of the solid components; but arteriovenous shunting may occur. Although well circumscribed, a true capsule is not usually present. Although extremely rare, there are a few example of intratumoural calcification with $\mathrm{MH}$. Calcification is more often seen in other hepatic tumours than in $\mathrm{MH}$ [8]. A solid variant of $\mathrm{MH}$ is occasionally seen but it is usually smaller in size [9].

MRI features of MH are still emerging. MRI reflects the underlying pathology, exquisitely demonstrates the cystic and solid components and identifies the sharp outlines of the lesion separate from rest of the tissues. Owing to its massive size, $\mathrm{MH}$ may have a huge exophytic component closely abutting the extrahepatic solid organs. In such instances MRI enables confident confirmation of its hepatic origin. The cystic component of $\mathrm{MH}$ may demonstrate varying signal intensity consistent with the concentration of proteins within [10]. The poor enhancement of the solid components of the tumour in the arterial, portovenous as well as delayed phases of dynamic post-gadolinium study, seen in this patient, was unlike the typical enhancement patterns identified with hepatocellular carcinoma, cystic hepatoblastoma or haemangioma. If this pattern is consistently observed in more patients, a dynamic postcontrast MRI may be regularly recommended in the evaluation of $\mathrm{MH}$. The relationship of the mass with the intrahepatic vasculature and status of the biliary tree are also best demonstrated on MRI. 
A cystic MH may need to be distinguished from several other cystic and complex solid-cystic lesions of the liver such as congenital liver cysts, hydatid cyst, liver abscesses, cavernous haemangiomas and haemangioendotheliomas, teratomas, hepatoblastomas, hepatocellular carcinomas and embryonal sarcomas. In polycystic liver disease the cysts are generally scattered in the entire liver and 50\% have cysts in spleen and kidneys. Hepatic abscess shows clinical and haematological evidence of sepsis. Amoebic abscess needs to be excluded in endemic zones. The several complex forms of its presentation makes hydatid disease a mimic for multiple cystic conditions. The collagen-rich pericyst of hydatid cyst is hypointense on all MR sequences and its enhancement is restricted to the periphery. It takes several years for a hydatid cyst to enlarge enough to reach the average size of a cystic $\mathrm{MH}$, hence an unlikely occurrence in infants. The enhancement properties of a large cavernous haemangioma may reveal its identity at dynamic CT or MRI, although the differentiation may be sometimes difficult [10]. Teratomas usually contain calcium and fat. Intratumoural calcification is present in $50 \%$ of hepatoblastomas and $40 \%$ of haemangioendotheliomas [11]. Both of these tumours are predominantly solid, with the latter associated with high output cardiac failure, bruit over the liver and marked hypervascularity [11]. Cystic hepatoblastoma is very rare and tends to show early arterial enhancement and vascular invasion [10, 11]. Undifferentiated embryonal sarcomas that are hypovascular with myxoid filled cystic areas may be difficult to distinguish from a cystic $\mathrm{MH}$; but they are even rarer and usually seen in older children [12].

In the aforementioned patient the massive size of the tumour was a hindrance in surgical excision and increased the technical challenge in its removal. This problem was circumvented through the on-table ultrasound-guided aspiration of fluid from the cystic components of the mass that reduced the tumour volume and improved the access for surgical dissection. Moreover, despite all the pre-operative imaging studies the intrahepatic vessels were suboptimally assessed since they were distorted and effaced by the mass effect of the large tumour. However, post-decompression of the cysts, aspiration decreased the mass effect significantly and a clear intervening plane between the margins of the lesion and the intrahepatic veins became apparent. This enabled confident resection without injuring the vessels. This simple procedure of ultrasound-guided aspiration of the cystic components expanded the role of the radiologist in patient management beyond routine diagnostic imaging and image-guided biopsy. We look upon this as an example of radiology broadening its horizons in patient management through pro-active participation.

\section{References}

1. Ros P, Goodman Z, Ishak K, Dachman AH, Olmstead WW, Hartman D S, et al. Mesenchymal hamartoma of the liver: radiologic-pathologic correlation. Radiology 1986;158: 619-24.

2. Stocker JT, Ishak KG. Mesenchymal hamartoma of the liver: report of 30 cases and review of the literature. Pediatr Pathol 1983;1:245-67.

3. Weinberg AG, Finegold MJ. Primary hepatic tumours of childhood. Hum Pathol 1983;14:512-37.

4. Stringer MD, Alizau NK. Mesenchymal hamartoma of the liver: a systematic review. J Pediatric Surg 2005;40: 1681-90.

5. Murray JD, Ricketts RR. Mesenchymal hamartoma of the liver. Am Surg 1998;64:1097-103.

6. Karpelowsky JS, Pansini A, Lazarus C, Rode H, Millar AJW. Difficulties in the management of mesenchymal hamartomas. Pediatr Surg Int 2008;24:1171-5.

7. Stanley P, Hall TR, Woolley MM, Diament MJ, Gilsanz V, Miller JH. Mesenchymal hamartomas of the liver in childhood: Sonographic and CT findings. AJR Am J Roentgenol 1986;147:1035-9.

8. Steiner M, Giles H. Mesenchymal hamartoma of the liver demonstrating peripheral calcifications in a 12-year-old boy. Pediatr Radiol 2008;38:1232-4.

9. Koumanidou C, Vakaki M, Papadaki M, Pitsoulakis G, Savvidou D, Kakavakis K. New sonographic appearance of hepatic mesenchymal hamartoma in childhood. J Clin Ultrasound 1999;17:164-7.

10. Powers C, Ros PR, Stoupis C, Johnson WK, Segel K. Primary liver neoplasms: MR imaging with pathologic correlations. Radiographics 1994;14:459-82.

11. Helmberger TK, Ros PR, Mergo PJ, Tomezak R, Reiser MF. Pediatric liver neoplasms: a radiologic-pathologic correlation. Eur Radiol 1999;9:1339-47.

12. Moon WK, Kim WS, Kim IO Yeon KM, Yu IK, Choi BI, et al. Undifferentiated embryonal sarcoma of the liver: US and CT findings. Pediatr Radiol 1994;24:500-3. 